\title{
Linx
}

Revue des linguistes de l'université Paris X Nanterre

$12 \mid 2002$

"Comme la lettre dit la vie »

\section{Promesses et anaphores}

\section{Marielle Lignereux}

\section{OpenEdition}

\section{Journals}

Édition électronique

URL : http://journals.openedition.org/linx/1295

DOI : 10.4000/linx.1295

ISSN : 2118-9692

\section{Éditeur}

Presses universitaires de Paris Nanterre

\section{Édition imprimée}

Date de publication : 1 octobre 2002

Pagination : 140-148

ISSN : 0246-8743

\section{Référence électronique}

Marielle Lignereux, "Promesses et anaphores », Linx [En ligne], 12 | 2002, mis en ligne le 10 octobre 2012, consulté le 30 avril 2019. URL : http://journals.openedition.org/linx/1295 ; DOI : 10.4000/ linx. 1295

Ce document a été généré automatiquement le 30 avril 2019.

Département de Sciences du langage, Université Paris Ouest 


\title{
Promesses et anaphores
}

\author{
Marielle Lignereux
}

1 Dans le cadre d'une réflexion plus large sur les verbes d'engagement, il était nécessaire de mieux comprendre le fonctionnement et le sens précis de chaque verbe de promesse. Autant que l'on puisse interpréter les exemples complexes de notre corpus constitué d'extraits de la littérature des $\mathrm{XII}^{\mathrm{e}}$ et XIII ${ }^{\mathrm{e}}$ siècles, de légères différences apparaissaient au sein d'un champ commun.

2 Puisqu'aucun locuteur ne peut gloser les exemples extraits de notre corpus, nous nous arrêterons sur les moyens que nous donne le texte. Nous observerons la ou les relations qu'ils entretiennent avec les substantifs et d'autres verbes d'engagement par exemple; ce qui nous permettra d'affiner nos premières conclusions fondées davantage sur l'intuition du locuteur moderne. Quels énoncés sont anaphorisés par quels verbes? Quels substantifs anaphorisent quel verbe? Comment, enfin, les verbes d'engagement (abrégés désormais en $V(E N G))$ eux-mêmes jouent-ils entre eux?

3 L'anaphore participe pleinement à la construction du sens et est l'un des éléments essentiels dans le processus d'interprétation.

\section{L'anaphore d'énoncés non-marqués}

4 Nous nous arrêterons en premier lieu sur un type d'emploi particulier des V(ENG). Dans notre corpus en effet, nous avons repéré des cas où V(ENG) anaphorise un énoncé nonmarqué, que nous noterons $\mathbf{E}(\mathbf{0})$. Nous entendons par énoncé non-marqué un énoncé qui ne comprend pas de marques illocutoires explicites. Nos exemples présentent en majorité des anaphores par V(ENG) de $\mathrm{E}(0)$ au futur ; que nous noterons désormais ainsi $\mathbf{E}$ (futur).

5 Comme fondement de notre réflexion, nous prendrons trois extraits d'Erec qui présentent la particularité de reprendre par trois fois le verbe promettre:

(1) Quant li beisiers del cerffu pris

A la costume del païs,

Erec, come cortois et frans

$\mathrm{Fu}$ de son povre oste an espans:

De ce que promis li avoitCovant mantir ne li voloit. (Erec 1 , v. 1797 - 1802) 
Quand le baiser du cerf eut été pris, selon la coutume du pays, Erec, en homme courtois et loyal, se préoccupa de son hôte peu fortuné : il ne voulait pas manquer à la promesse qu'il avait faite. (trad. R. Louis)

(2) Deus chastiax lor avoit promis,

Les plus biax et les mialz asis,

Et ces qui mains dotoient guerre

Qui fussent an tote la terre (Erec, v. 1829 - 1832)

Il leur avait promis deux châteaux, les plus beaux et les mieux situés de tout le pays, et ceux qui redoutaient le moins la guerre. (trad. R. Louis)

(3) Quant an son roiaume vandroient,

Ces deus chastiax lor liverroient,

Et les rantes et les justises,

Si com il lor avoit promises.(Erec, v., 1835 - 1838)

Une fois arrivés en son royaume, ils leur livreraient ces deux châteaux avec les rentes et les droits de justice, ainsi qu'il le leur avait promis. (trad. R. Louis)

6 C'est le narrateur qui emploie ici les trois occurrences de promettre. Le niveau d'énonciation est donc modifié : le narrateur rapporte les propos d'Erec. Les occurrences de promettre sont donc ici au discours rapporté mais l'énoncé initial d'Erec est au discours direct:

(4) Iluec vos donrai deus chastiax,

Molt boens, molt riches, et molt biax;

Sires seroiz de Roadan,

Qui fu fez des le tans Adan,

Et d'un autre chastel selonc

Qui ne valt mie moins un jonc;

La gent l'apelent Montrevel,

Mes peres n'a meillor chastel. (Erec, v. 1317 - 1324)

Là, je vous donnerai deux châteaux, très bien bâtis, très puissants et très beaux ; vous serez sire de Roadan, qui fut construit dès le temps d'Adam, et d'un autre château voisin, qui ne lui cède pas en valeur du prix d'un jonc: les gens l'appellent Montrevel, mon père n'a pas de meilleur château. (trad. R. Louis)

Si l'on observe attentivement le discours d'Erec, on remarque qu'il n'emploie aucun verbe d'engagement; il ne marque pas son discours. On repère seulement qu'il utilise le verbe donner au futur. Le verbe promettre anaphorise donc un énoncé $\mathrm{E}(0)$ au futur.

7 Pour expliquer cette anaphore, nous pouvons avancer deux hypothèses. La première expliquerait l'absence de verbe par le statut de l'énonciateur. Le statut de l'énonciateur en effet, surtout lorsqu'il exerce une fonction d'autorité, est en soi une marque d'engagement, une garantie. Alors, étant donné la fonction de futur roi d'Erec, son rang, sa lignée, il n'est pas nécessaire qu'il marque formellement son discours. La seconde hypothèse tendrait à prouver que certains énoncés non-marqués au futur seraient des énoncés de promesse. Le verbe promettre serait donc un indice d'interprétation a posteriori de $E$ (futur).

Un autre exemple confirme encore les deux hypothèses avancées supra :

(5) Dedenz celui terme avint que li apostoiles de Rome sot que li rois Artus avoit sa fame

lessiee et qu'il prometoit qu'il l'ocirroit, s'il la pooit tenir; (Mort Artu' ${ }^{2}$, p. 117)

Pendant ce temps, il arriva que le pape de Rome apprit que le roi Arthur avait quitté

sa femme et promettait de la tuer, s'il pouvait la saisir. (trad. M.L. Ollier)

A nouveau, promettre anaphorise E (futur) car si nous nous reportons au passage au discours direct où le roi Arthur s'engage, nous remarquons que seul le futur marque son engagement (si morra ele) : 
(6) -Je bé, fet li rois, que por ce mesfet qu'ele a fet l'en en face grant justise. Et ge vos commant, fet il, tout premierement, por ce que vos estes rois, et as autres barons, qui ceanz sont, aprés, et si le vos requier seur le serement que vos m'avez fet, que vos esgardoiz entre vos de quel mort ele doit morir; que sanz mort n'en puet ele eschaper, se vos meïsmes vos teniez devers lui, en tel maniere que, se vos disiez qu'ele ne deüst pas morir, si morra ele. ( Mort Artu, p. 92)

Je veux, dit le roi, qu'elle soit durement châtiée pour le crime qu'elle a commis. Et, ajouta-t-il, je vous demande à vous d'abord, en votre qualité de roi, et aux autres barons ici présents ensuite, en invoquant le serment que vous m'avez prêté, de décider entre vous de quelle mort elle doit mourir ; car elle ne peut échapper à la mort, dussiez-vous vous ranger de son côté, au point même que si vous exprimiez un avis contraire, elle mourra néanmoins. (trad. M-L. Ollier)

L'hypothèse du statut de l'énonciateur est sans conteste un élément déterminant. Cependant, nous pouvons citer des exemples où l'engagement n'est pas marqué et où le statut de l'énonciateur n'a guère d'influence. Et parmi les occurrences relevées ${ }^{3}$, nous retiendrons un extrait de la Queste ${ }^{4}$ p. 30 :

(7) «Tant m'en as conjuré, fet li chevaliers, que je le te dirai. Mes ce ne sera mie a toi seul, ainz vuel que tu i amoines le chevalier a qui tu porteras l'escu. "

- Tu me le demandes avec tant d'insistance, répond le chevalier, que je te le dirai, mais pas seulement à toi. Je veux en effet que tu fasses également venir ici le chevalier à qui tu remettras l'écu. (trad. E. Baumgartner)

Le chevalier joue ici un rôle secondaire dans le récit. Et pourtant, nous retrouvons la même structure : aucun verbe d'engagement n'est exprimé ici, si ce n'est la présence du futur je le te dirai. Mais dans la suite du texte p. 30 - 31, V(ENG) apparaît pour reprendre E (0) « car einsi le m'a il promis ». Il convient donc de relire $\mathrm{E}(0)$ comme un énoncé marqué.

9 A la lecture de cette occurrence, il est impossible d'avancer systématiquement l'hypothèse du rôle joué par le statut de l'énonciateur. Dans certains cas, ce rôle est incontestable. En revanche, la seconde hypothèse selon laquelle un énoncé non-marqué est anaphorisé par un verbe de promesse demeure une constante. Nous pouvons donc en déduire que nous sommes face à une valeur particulière du futur; ce que nous développerons par ailleurs.

Toutefois, on pourrait nous rétorquer que, dans ce cas, l'opinion du narrateur est en cause car il transposerait l'énoncé du locuteur, et l'interpréterait, peut-être de manière fautive. Mais il se trouve que notre corpus comprend un exemple où le même locuteur, l'Orgueilleux de la Lande, prononce les deux énoncés suivants :

(8) « Molt me puet an irié veoir.

Qant je reving et je lo soi,

Si jurai molt, et droit an oi,

Que d'avoine ne mangeroit

Ses palefroiz ne ne seroit

Saigniez ne ferrez de novel,

Ne n'avroit cote ne mantel

Autre qu'ele avoit a cele eure,

Tant que je venise au deseure

e celui qui l'ot esforciee,

Et mort et la teste tranchiee. " (Conte du Graal ${ }^{5} \mathrm{v} .3822-3832$ )

On put voir quelle était ma colère à mon retour, quand je l'appris. Je fis le serment solennel, et avec raison, que son palefroi n'aurait pas d'avoine à manger, qu'il ne serait pas saigné ni ferré de nouveau, et qu'elle-même n'aurait pas de tunique ni de manteau autres que ceux qu'elle portait à cette heure, avant que je n'aie eu le dessus sur celui qui l'avait violentée, que je l'aie tué et que je lui aie tranché la tête. (trad. C. Méla) 
(9) « Entree estes en male voie,

Entree estes en male paine,

Ne ja ne mangera d'avaine

Vostre chevaus, ne n'iert ferrez

Tant que cil estera tuez,

Et la o il desferrera

amais referrez ne sera.

S'i[l] muert, vos me sivroiz a pié

Ne ja ne seront changié

Li drap que vos avez vestue,

Ainz me sivrez a pié et nue

Tant que la teste en avrai prise,

Ja n'en ferai autre jostise. " (Conte du Graal, v. 778-790)

Mais vous voilà dans une mauvaise passe, avec bien des tourments en cours de route! Je vous le jure ${ }^{6}$, votre cheval ne mangera pas d'avoine, ne sera pas saigné avant que je me sois vengé, et, s'il vient à perdre ses fers, il ne sera pas referré. S'il meurt, vous me suivrez à pied. Jamais non plus vous ne changerez les vêtements que vous portez. Vous finirez par me suivre nue et à pied et cela jusqu'à ce que je lui aie tranché la tête : c'est la justice que je prendrai de lui. (trad. C. Méla)

Dans ce cas donc, le narrateur n'intervient pas : le locuteur glose lui-même son énoncé en rendant explicite la valeur de son discours : c'est bien un serment qu'il a fait, si l'on en croit le verbe jurer.

13 Nous retiendrons dans un premier temps que le statut de l'énonciateur, lorsqu'il occupe une position élévée dans la hiérarchie sociale, le dispense de s'engager formellement en employant un V(ENG). Cette approche est incontestable lorsqu'elle concerne Erec, Gauvain, Arthur... Lorsque ce n'est pas le cas, c'est sur la valeur du futur qu'il convient de se pencher, tout en sachant que la première hypothèse n'exclut pas la seconde. Nous garderons en mémoire que dans notre corpus, certains verbes d'engagement, promettre, jurer mais aussi creanter, lorsqu'ils anaphorisent E (futur), rendent explicite la force illocutoire de promesse. $\mathrm{V}(\mathrm{ENG})$ n'est peut-être qu'un outil dans le processus d'interprétation.

\section{Les substantifs}

Une autre approche possible consiste à relever et à analyser les substantifs car un substantif peut soit apporter un commentaire ou bien un élément d'interprétation soit anaphoriser un énoncé.

Parmi les substantifs attestés dans notre corpus, deux cas de figure se présentent. En premier lieu, le substantif peut aussi anaphoriser un énoncé non-marqué. C'est le cas au paragraphe 91 de la Queste et nous citerons par exemple :

(10) Quant Lancelot vit le chevalier venir, si ne corut mie prendre ses armes, come cil qui bien pensoit que ce estoit la promesse que li preudons li avoit fete de Galaad, qui seroit o lui et li feroit compaignie une piece de tens. (Queste p. 250)

Lancelot, en le voyant approcher, ne courut pas prendre ses armes tant il était persuadé que c'était là Galaad, le compagnon que lui avait promis l'ermite. (trad. E. Baumgartner)

Le substantif promesse anaphorise l'énoncé suivant : 
(11) Mes ançois qu'il [li prudons] se partist de la roche comença il a huchier : " Ha! Lancelot, serjanz Jhesucrist, por Dieu ne m'oublie pas, mes prie Galaad, le verai chevalier, que tu avras par tens o toi, qu'il prit a Nostre Seignor qu'Il par sa douce pitié ait de moi merci!» Einsi crioit li preudons aprés Lancelot, qui molt ert liez de la novele que cil li ot dite, que Galaad devoit estre prochainnement ses compainz. (Queste p. 249)

Mais, avant de s'en aller, il crie encore à Lancelot : " Ha! Lancelot, soldat du Christ, au nom de Dieu ne m'oublie pas mais demande à Galaad, le vrai chevalier que tu vas bientôt retrouver, de prier Notre Seigneur d'avoir pitié de moi dans sa miséricorde ". Lancelot cependant se réjouissait de savoir qu'il allait bientôt revoir Galaad. (trad. E. Baumgartner)

17 En second lieu, nous nous attarderons sur les occurrences où le substantif promesse anaphorise un engagement marqué par le verbe promettre comme le prouve l'exemple suivant :

(12) Vos savez bien que Jhesucriz fu entre ses apostres pastres et mestres a la table de la Ceinne; aprés fu senefiee par Joseph la Table del Saint Graal, et la Table Reonde par cest chevalier. Nostre sires promist a ses apostres devant sa Passion que il les vendroit visiter et veoir, et il s'atendirent a ceste promesse triste et esmaié.

(Queste p.78)

Jésus-Christ, vous le savez, apparut à la table de la Cène comme le pasteur et le maître de ses apôtres. De même Joseph donna-t-il sa signification à la table du Saint-Graal et le chevalier dont nous parlons à la Table Ronde. Avant sa Passion, Jésus-Christ promit à ses apôtres qu'Il viendrait les visiter et ils attendirent dans la tristesse et l'anxiété l'accomplissement de la promesse. (trad. E. Baumgartner)

Ou encore :

(13) Cil li distrent la verité,

comant il estoit avenu

de son pere le viel chenu,

qui morz estoit et trespassez.

Erec an pesa plus asez

qu'il ne mostra sanblant as genz,

mes diaus de roi n'est mie genz,

n'a roi n'avient qu'il face duel.

La ou il ert, a Tintajuel,

fist chanter vigiles et messes,

promist et randi les promesses,

si com il les avoit promises,

as meisons Deu et as eglises. (Erec v. 6462-6474)

Ils lui dirent la vérité : comment son père, le vieillard aux cheveux blancs, était mort et trépassé. Erec eut beaucoup plus de peine qu'il n'en laissa paraître à ses gens; mais deuil de roi n'est pas de bon ton et il ne convient pas qu'un roi manifeste sa peine. Là où il se trouvait à Tintagel, il fit chanter des vigiles et des messes, fit des promesses aux maisons-Dieu et aux églises et s'en acquitta comme il s'y était engagé. (trad. R. Louis)

Dire que le substantif promesse anaphorise le verbe promettre relève de la tautologie, mais il fallait s'assurer que le substantif promesse en ancien français anaphorisait bien un engagement. Nous avons relevé aussi un exemple où le substantif renvoie à un verbe d'engagement autre que promettre :

(14) Endementeirs qu'il aloit chevalchant par ses viles et sejornant de jor en jor par ses chastiax la ou il les savoit muez aiesiez, l'amonesta tant messires Gauvains qu'il recomençast la guerre encontre Lancelot qu'il li acreanta come rois que, ja plus tost la Pasque ne seroit passee, qu'il iroit a ost banie seur Lancelot et tant se traveilleroit, s'il i devoit morir, qu'il abatroit les fortereces de Banoïc et de Gaunes en tel maniere qu'il ne leroit en mur pierre seur autre. Ceste promesse fist li rois a monseigneur Gauvain; si li promist ce qu'il ne li pot mie tenir. (Mort Artu, 128) 
Pendant qu'il parcourait à cheval ses villes, séjournant au fil des jours dans ceux de ses châteaux qu'il savait les plus confortables, messire Gauvain le pressa tant de reprendre la guerre contre Lancelot qu'il lui donna sa parole de roi : sitôt passée la fête de Pâques, il convoquerait toute son armée pour aller contre Lancelot, et mettrait tout en œuvre, dût-il y mourir, pour abattre les forteresses de Benoÿc et de Gaunes sans y laisser pierre sur pierre. C'est la promesse que le roi fit à messire Gauvain; il lui promit ainsi ce qu'il ne fut pas en mesure de tenir. (trad. M-L. Ollier) Nous pouvons donc supposer que le verbe (a)creanter, verbe d'engagement faible, associé au renforcement de l'engagement indiquant le statut de l'énonciateur équivaut à une promesse.

19 Se dessine progressivement un classement adéquat pour les verbes de promesse; les substantifs se révèlent de bons instruments et confortent les hypothèses établies.

\section{Un verbe de promesse anaphorise un autre verbe de promesse}

La question de l'anaphore d'un verbe d'engagement par un verbe d'engagement se pose à présent. Dans certains cas, le texte ne nous aide pas à classer les verbes puisque V(ENG) anaphorise le même verbe d'engagement. Nous n'insisterons pas sur ce point. En revanche, nous nous attarderons maintenant sur les cas où un verbe d'engagement V2 (ENG) anaphorise un autre verbe d'engagement V1 (ENG). Les verbes (a)creanter, afier, fianc(i)er, jurer, plevir et promettre, parce qu'ils appartiennent au même champ, tissent entre eux des liens que l'on peut supposer d'antonymie ou de synonymie. Nous nous arrêterons d'abord sur les exemples où le texte lui-même glose les occurrences des verbes d'engagement.

\section{1. promettre et (a)creanter}

Nous avons vu que le substantif promesse anaphorisait un engagement marqué par le verbe (a)creanter. Nous constatons que le verbe promettre anaphorise aussi (a)creanter :

(15) Endementeirs qu'il aloit chevalchant par ses viles et sejornant de jor en jor par ses chastiax la ou il les savoit muez aiesiez, l'amonesta tant messires Gauvains qu'il recomençast la guerre encontre Lancelot qu'il li acreanta come rois que, ja plus tost la Pasque ne seroit passee, qu'il iroit a ost banie seur Lancelot et tant se traveilleroit, s'il i devoit morir, qu'il abatroit les fortereces de Banoïc et de Gaunes en tel maniere qu'il ne leroit en mur pierre seur autre. Ceste promesse fist li rois a monseigneur Gauvain; si li promist ce qu'il ne li pot mie tenir. (Mort Artu, 128)

Pendant qu'il parcourait à cheval ses villes, séjournant au fil des jours dans ceux de ses châteaux qu'il savait les plus confortables, messire Gauvain le pressa tant de reprendre la guerre contre Lancelot qu'il lui donna sa parole de roi : sitôt passée la fête de Pâques, il convoquerait toute son armée pour aller contre Lancelot, et mettrait tout en oeuvre, dût-il y mourir, pour abattre les forteresses de Benoÿc et de Gaunes sans y laisser pierre sur pierre. C'est la promesse que le roi fit à messire Gauvain; il lui promit ainsi ce qu'il ne fut pas en mesure de tenir. (trad. M-L. Ollier)

L'anaphore ici est intéressante à double titre: l'énoncé marqué par acreanter est anaphorisé en premier lieu par le substantif promesse, puis en second lieu par le verbe promettre. 
23 Nous avons démontré qu'il n'est pas nécessaire pour un personnage exerçant une fonction d'autorité de s'engager fortement. Il suffit qu'il s'engage comme l'indique le verbe acreanter et la qualité de roi explicite ici supplée les garanties nécessaires.

\section{2. promettre et plevir}

Nous avons relevé deux occurrences où le verbe promettre anaphorise le verbe plevir. Nous citerons un extrait d'Erec:

(16) -Sire, fet il,je vos plevis

Que, ja tant con je soie vis,

N'avroiz de mon secors mestier

Que tantost ne vos vaigne aidier

A quanque je porrai mander.

-Ja plus ne vos quier demander,

Fet Erec, molt m'avez promis ;

Mes sire estes et mes amis,

Se l'uevre est tex con la parole. » (Erec, v. 3891 - 3899)

- Sire, fait il, je vous promets que, tant que je vivrai, chaque fois que vous aurez besoin de mon aide, j'irai aussitôt vous secourir avec tous les gens que je pourrai assembler. - Je n'ai rien de plus à vous demander, fait Erec, c'est déjà beaucoup promettre. Vous êtes mon seigneur et mon ami si vos actes répondent à vos paroles. (trad. R. Louis)

Avec cet exemple, on est en droit de se demander si promettre anaphorise l'engagement en soi, je vos plevis, ou s'il ne reprend pas plutôt le contenu propositionnel de la promesse ja tant con je soie vis, N'avroiz de mon secors mestier Que tantost ne vos vaigne aidier A quanque je porrai mander.

En effet, le verbe promettre ne renvoie pas exclusivement à l'énonciation de plevir mais aussi au contenu propositionnel. Le verbe plevir marque une forme d'engagement mais nous constatons que ce n'est pas le verbe seul qui fait l'objet de l'attention de l'allocutaire. L'adverbe molt est ici la clé de la solution car c'est l'adverbe qui oriente l'interprétation : promettre porte aussi sur le contenu de l'engagement, sur l'enjeu de la promesse.

\section{3. promettre et afier}

27 Nous avons relevé dans notre corpus un cas où le verbe promettre anaphorise un engagement marqué par le verbe afier :

(17) Lors le fist li rois amener, en son estant le fist lever.

«Aï, fait il, rous de pute aire, tant par ies or de mal afaire

et si es male criasture.

Certes en toi pecha Nature, qant tu ne puez a bien entendre, filz a putain, dines de pandre." Devant le roi fu en estant Renart, si dist an soupirant :

« Ha! gentis rois, por Dieu merci; tenez ma foi :je vos afi ja mes clamor n'orez de moi, jel vos promest en bone foi. (Rom. Ren ${ }^{7}$., v. 1451-1464) 
Il le fait amener devant lui, le fait mettre debout, et dit : « Ah ! rouquin, engeance détestable, race maudite, créature perverse! Nature a commis une faute en te donnant la vie, toi qui ne peux suivre le droit chemin, fils de garce, pebdard!» Renart se tient debout devant le roi ; il dit en soupirant: « Hélas, roi magnanime, grâce, au nom de Dieu! Vous avez ma parole : je vous jure que jamais plus vous n'entendrez personne se plaindre de moi : je vous le promets loyalement. (trad.H. Rey-Flaud et A. Eskénazi)

Devant cet exemple, nous pourrions adopter la même réserve qu'en (16) : le verbe promettre porte-t-il sur le verbe afier ou plutôt sur le contenu propositionnel ? Il semble bien que le verbe promettre anaphorise davantage le contenu propositionnel puisque promettre est construit avec un COD qui explicite l'anaphore : le (puisque jel est une enclise constituée de je + le).

\section{4. fianc(i)er et plevir}

Dans notre corpus, le verbe plevir anaphorise un engagement dans la majorité des cas marqué par fianc(i)er; et cela à 4 reprises. Dans ce cas, nous pouvons avancer l'hypothèse de la synonymie entre ces deux verbes avec plus d'assurance. C'est par exemple :

(18) Et lors dit a Morgain: "Bele suer, ge vos pri que vos me diez verité de ce que ge vos demanderai. » Et ele respont que si fera ele volentiers, se ele le set. "Fianciez le moi ", fet li rois; et ele li fiance. "Or vos requier ge, fet li rois, par la foi que vos me devez et que vos m'avez ici plevie, que vos me diez qui ces ymages portrest, se vos en savez la verité, et nel lestiez por nule chose. (Mort Artu, 52)

Il s'adressa alors à Morgue : "Chère soeur, je vous prie de me dire la vérité sur ce que je vais vous demander.» Elle lui assura qu'elle le ferait volontiers, si elle la connaissait. "Promettez-le moi », dit le roi ; elle le lui promit. "A présent je vous somme, par la foi que vous me devez et que vous venez d'engager par votre promesse, de me dire qui a peint ces images, si vous savez ce qu'il en est, et de ne vous y dérober sous aucun prétexte ». (trad. M.L.Ollier)

Cet exemple est intéressant à double titre. D'une part, on constate qu'à la demande d'Arthur de dire la vérité, Morgue répond sans employer un quelconque verbe d'engagement: ele respont que si fera ele volentiers, se ele le set. Elle utilise seulement le futur; ce qui a priori est insuffisant puisque le roi Arthur demande qu'elle marque son engagement en gageant sa parole; c'est pourquoi Arthur insiste : "Fianciez le moi », fet li rois. D'autre part, le contexte droit commente le verbe fiancier ainsi que ce qu'il implique : on engage ainsi sa parole, sa foi, i.e. sa parole d'honneur.

C'est aussi le cas dans le cadre d'un engagement spécifique. Après un combat, le chevalier vaincu doit obéir aux injonctions du chevalier vainqueur. Dans cet exemple, le Bel Inconnu demande à Giflet de se rendre auprès du roi Arthur :

(19) Mais cil, qui desous lui le tient,

Li dist : «Sire, el i covient ;

A tant n'en irés vos plus cuites,

Vostre estre et vostre non me dites,

Et si fiancerés prison

Que vos irés sans oquison

Ens en la cort Artus le roi."

Li chevaliers plevisa foi,

Puis li a dit aprés son non:

"Sire, Giflet m'apele on;

Giflés, li fius Do, sui nonmés

En cest païs et apielés. 
Vostre sui tos d'or en avant,

Car molt vos sa prué et vaillant. » (Bel Inc ${ }^{8}$. v. 1795-1808)

Mais notre héros, tout en le maintenant sous lui, répondit : « Seigneur, ce n'est pas tout, vous n'en serez pas quitte pour autant : dites-moi votre nom, qui vous êtes, et jurez-moi que vous irez sans tarder vous constituer prisonnier à la cour du roi Arthur. » Le chevalier donna sa parole, puis il révéla son nom : "Seigneur, mon nom est Giglet ; Giflet, fils de Do, c'est ainsi qu'on m'appelle et me nomme dans ce pays. Je me rends à vous aujourd'hui, je reconnais votre valeur et votre courage. » (trad. M. Perret et I. Weill)

Enfin, nous citerons un exemple extrait d'un serment solennel :

(20) mes tant com il avra la guerre

et l'ire et le mal vers sa dame,

n'a en cest mont home ne fame

cui il suiest, mien escïant,

tant que il li jurt et fiant

qu'il fera tote sa puissance

de racorder la mescheance

que sa dame a si grant a lui

qu'il an muert de duel et d'enui. »

Et la dame dit: " Je sui preste,

einz que vos entroiz an la queste,

que je vos plevisse ma foi

et jurerai, s'il vient a moi,

que je, sanz guile et sanz feintise,

li ferai tot a sa devise

sa pes, se je feire la puis. » (Yvain ${ }^{9}$, v. 6596-6611)

Mais tant qu'il sera en guerre contre sa dame et tant que la colère et le ressentiment habiteront celle-ci, il ne daignera suivre personne, ni homme ni femme, en ce bas monde. Il faudrait d'abord lui jurer de faire l'impossible pour mettre fin à sa disgrâce auprès de sa dame, car cette disgrâce l'accable de douleur et de tourment. - «Avant de vous voir partir à sa recherche, lui répond la dame, je suis prête à vous promettre et à vous jurer que, s'il vient à moi, je lui procurerai, sans ruse et sans arrière-pensée, la paix qu'il souhaitera, si du moins je le puis. » (trad. P. Walter)

Dans cet exemple, le verbe jurer anaphorise le verbe jurer, en revanche c'est l'expression plevir sa foi qui anaphorise le verbe fianc(i)er.

\section{5. jurer et plevir en main}

31 Nous avons relevé une seule occurrence où jurer est anaphorisé par plevir en main :

(21) Li hermite Tristan connut,

Sor sa potence apoié fu ;

Aresne le, oiez conment:

"Sire Tristan, grant soirement

A l'en juré par Cornoualle,

Qui vos rendroit au roi, sanz falle

Cent mars avroit a gerredon.

En ceste terre n'a baron

Au roi ne l'ait plevi en main,

Vos rendre a lui o mort ou sain. » (Béroul, Tristan ${ }^{10}$, v. 1367-1376)

L'ermite reconnut Tristan. Appuyé sur sa canne, il lui dit: "Sire Tristan, toute la Cornouailles s'est engagée solennellement. Celui qui vous livrera au roi sans faute recevra cent marcs de récompense ". Tous les barons de ce pays ont donc juré au roi, la main dans celle de Marc, de vous livrer à lui, mort ou vif. (trad. P. Walter) 
Nous résumerons sous forme de tableau les liens qui existent entre les différents verbes d'engagement que nous avons particulièrement étudiés :

\begin{tabular}{|l|l|l|l|l|l|l|}
\hline anaphorisé par 䣲 & AFIER & (A)CREANTER & FIANC(I)ER & JURER & PLEVIR & PROMETTRE \\
\hline Promettre & - & + & - & - & - & - \\
\hline Plevir & - & - & + & - & - & - \\
\hline Plevir en main & - & - & - & + & - & - \\
\hline
\end{tabular}

Nous n'intégrons pas dans ce tableau les occurrences de promettre et plevir, de même que promettre + afier puisque l'analyse a montré que dans les deux cas, promettre anaphorisait le contenu propositionnel et non le verbe d'engagement.

L'anaphore permet donc de mettre en évidence le sème commun entre promettre et plevir, promettre et creanter, promettre et afier, fiancer et plevir sa foi. En revanche, jurer ne se laisse pas anaphoriser par un autre verbe simple : seul un, plevir en main, où le GNprép renforce la valeur du verbe, peut prendre la force d'engagement de jurer.

Nous avons mis en valeur que deux verbes, promettre et plevir, anaphorisent des engagements marqués par d'autres verbes (creanter pour promettre, fianc(i)er pour plevir). L'anaphore est donc un élément indispensable pour effectuer un premier classement des verbes de promesse.

Toutefois, les autres $\mathrm{V}(\mathrm{ENG})$ marquent assurément un engagement mais notre corpus ne présente pas d'exemples d'anaphore qui nous permettraient d'établir un réseau de relations plus complexe ou plus fin; ce qui nous aiderait à construire plus précisément encore le sens de ces verbes.

\section{NOTES}

1. Chrétien De Troyes, Erec et Enide, éd. par M. Roques, Paris, Champion, 1981.

2. La Mort le roi Artu, éd. par J. Frappier, Paris, Champion, 1964.

3. C'est par exemple Raoul de Houdenc, v. 4637, Le roman de Renart, première branche, v. 608, v. 1464, Huon le Roi, v. 419, 420, 431, 441, Le conte du Graal, v. 4704, ou encore Béroul, le roman de Tristan, v. 2722.

4. La Queste del saint Graal, éd. par A. Pauphilet, Paris, Champion, 1984.

5. Chrétien De Troyes, Le Conte du Graal, éd. par C. Méla, Le livre de poche, coll. Lettres Gothiques, 1990.

6. On notera que le verbe jurer n'apparaît pas dans le texte.

7. Le roman de Renart, branche 1, éd. par M. Roques, Paris, Champion, 1982.

8. R. De Baujeu, Le bel Inconnu, éd. par G. Perrie Williams, Paris, Champion, 1983.

9. Chrétien De Troyes, Le chevalier au Lion (Yvain), éd. par M. Roques, Paris, Champion, 1982. 
10. Béroul, Le roman de Tristan, édité par P. Walter et D. Lacroix, Paris, Le livre de poche, coll. Lettres gothiques, 1989.

\section{AUTEUR}

MARIELLE LIGNEREUX

Université Paris-10 - MoDyCo 